\title{
The Berkeley Program on Molecules of Astrophysical Interest
}

\author{
Sumner P. Davis \\ Department of Physics University of California at Berkeley Berkeley, California 94720, \\ USA
}

\section{Introduction}

A systematic program of laboratory analyses of selected molecular spectra of astrophysical interest started in 1958 and continues to the present time. The program includes production of spectral atlases, tabulations of spectral lines, analyses, calculations of excitation energies and molecular parameters, measurements of radiative lifetimes, and determinations of transition strengths. Work has been completed or is in progress on the spectra of $\mathrm{ArH}^{+}, \mathrm{C}_{2}$, carbon clusters, $\mathrm{CN}, \mathrm{CS}, \mathrm{CaCl}, \mathrm{CaH}, \mathrm{CaS}, \mathrm{FeD}, \mathrm{FeH}, \mathrm{HgH}, \mathrm{HgD}$, InI, LaO, LaS, OD, OH, SH, $\mathrm{Si}_{2}, \mathrm{SiC}_{2}, \mathrm{TiCl}, \mathrm{TiO}, \mathrm{TiO}^{+}, \mathrm{VO}, \mathrm{YS}, \mathrm{ZrCl}, \mathrm{ZrO}$, and $\mathrm{ZrS}$. The basic needs for astronomically useful data have not changed, but laboratory and analysis methods have become more sophisticated in order to cope with ever greater demands for consistency, accuracy, and breadth of information. The Fourier transform spectrometer and computer codes for analyses have enhanced our ability to satisfy some of these demands.

The need for better laboratory spectra of astrophysically important molecules is a continuing one, no less important today than it was 35 years ago when the program at Berkeley was started. The genesis of the Berkeley program was at a meeting of the Joint Commission for Spectroscopy held in Columbus in 1956, where Charlotte Moore read a statement about molecular spectra of astrophysical interest. She proposed that a systematic program on the laboratory analyses of selected molecular spectra be planned immediately and carried out as soon as possible. Two years later at the Moscow meeting it was reported that a program directed by F.A. Jenkins and J.G. Phillips was being started in response to the well-defined need. In 1959 I joined the program and became a co-principal investigator in 1961 when Prof. Jenkins passed away (Phillips \& Davis 1962). Details of the program up to 1987 are given in Publications of the Astronomical Society of the Pacific (Davis 1987), which should be together with this for a complete picture. I'd like to mention here, a little bit of how we started and what we worked with, and then take you through the process of producing and analyzing a spectrum with our present instrumentation. The description will be abbreviated, but I hope you will end with an increased appreciation of the quality and scope of our results as well as their limitations. 


\title{
2 Early goals
}

\subsection{Scientific bibliography}

Right at the start it was recognized that an up-to-date bibliography and progress report of our work as well as others' were essential for keeping track of spectroscopy of astrophysical interest. The Berkeley Newsletter Analysis of Molecular Spectra was started and continues as a bimonthly publication. It is available to anyone for a modest subscription fee. In this newsletter appear references from about 150 journals, separated into categories of diatomic and small polyatomic species, and general information including theoretical studies. It is currently running about 450 references in each issue. The task of collecting references from current scientific journals is borne entirely by Dr. David M. Eakin, one of the early students, and for many years an award-winning high school science teacher. Cumulative files dating from 1986 are kept by Prof. Phillips, and data for particular molecules can be had for the asking. Complete back issues are not generally available. Fig. 1 is a reproduction of a recent title page.

\section{BERKELEY NEWSLETTER}

\section{ANALYSIS OF MOlECUlar SPECTRA}

\begin{abstract}
No. 200
July, 1992

You have undoubtedly noticed that this is Issue Number $\mathbf{2 0 0}$ of these bi-monthly Newsletters. It comes as quite a shock to us to realize that we have been distributing the Newsletters for over thirty three years. We are gratified that our 160 recipeints world-wide find it useful, and plan to continue its distribution as long as we are physically able to do so and as long as it proves to be of interest.
\end{abstract}

\section{BIBLIOGRAPHY}

$\mathrm{Al}_{2} \quad$ Electronic structure calculations of small $\mathrm{Al}_{\mathrm{n}}(\mathrm{n}=2-8)$ clusters

$\mathrm{Al}_{3} \quad$ T. Bastug et.al.

$\mathrm{Al}_{4} \quad$ Z. f. Phys. D 22, 641 (1992)

AlF The infrared emission spectrum of gaseous AlF

H.G. Hedderich and P.F. Bernath

J. Molec. Spectros. 153, 73 (1992)

AlH The ground-state infrared spectra of aluminum monodeuteride: the massindependent molecular parameters of group IIIa hydrides

R.D. Urban and H. Jones

Chem. Phys. Lett. 190, 609 (1992)

Fig. 1. Title page of the Berkeley Newsletter. 


\subsection{Spectrum Line Lists}

The original goal was simply to record an entire spectrum from $400 \mathrm{~nm}$ to 1200 $\mathrm{nm}$, analyze it, calculate molecular parameters, and provide a list of all identified lines. The spectrum chosen for the first one was the red system of CN covering the region from $480 \mathrm{~nm}$ to $1200 \mathrm{~nm}$, the long wavelength limit of photographic plates. Hundreds of plates were taken, tens of thousands of spectrum lines were measured semiautomatically, reduced by computer, analyzed, and finally tabulated band by band, branch by branch. At that time it was impractical to make a finding list. The final result was a book (Davis \& Phillips 1963). Work on the Swan system of $\mathrm{C}_{2}$ soon followed, and it too was incorporated into a book (Phillips \& Davis 1968). Subsequently book publishing became too expensive for long line lists. We settled for publication in the journals so far as possible.

\subsection{Atlases}

Spectrum atlases - plots of spectral intensity vs. either wavelength or wavenumber - are often more helpful than line lists when trying to identify the presence of particular species in stellar spectra, or to identify unknown spectral lines. We made enlargements of spectra for our own use and for publication, but the production of Gatterer et al.(1957) type of atlas seemed an unwise use of our time.

\subsection{Analysis and Calculation of Parameters}

Measurements of line positions must be separated into bands and branches and assigned to a specific rotational quantum number before useful parameters can be calculated. The line-by-line picking of branches soon became inadequate to separate complex overlapping structures and a more automated procedure was developed (Phillips 1959). He simply programmed a computer to do what he did by hand, and had it print out enough data for him to determine whether a branch was real or not. After that, parameter calculation was straightforward enough. However, line lists were still essential because using the parameters to reproduce the spectrum was not possible for everyone, since large computers were not readily available.

\subsection{Sources and Spectrometers}

Early spectroscopists exercised great ingenuity in constructing sources to coax unwary species to radiate and give up their secrets, but we used a few relatively simple ones. Our CN sources, for example, were an arc, a Geissler tube, and a King furnace.

The first spectrometer was a concave diffraction grating in a $6.3 \mathrm{~m}$ Paschen mounting which covered the spectrum up to $1200 \mathrm{~nm}$ in first order. The first grating was ruled by Rowland, and the second by Bausch and Lomb. The mounting dated back to the early 30 's, and was used by R. T. Birge in some of his early 
work. Later I obtained a nearly perfect original plane grating $13.5 \mathrm{~cm}$ wide (it was a generous gift from George Harrison at M.I.T. after he made six replica. He sent them out to silence his critics who scoffed at an interferometrically-controlled continuous-motion ruling engine). It had no ghosts of any significance and produced nearly symmetrical lines, even for those of high intensity. We installed it in a Czerny-Turner mounting with 3-meter focal length mirrors. It served us well for many years, and it proved to be superior in throughput, resolution, line-shape, and dispersion to the concave grating.

\subsection{Wavelength Standards}

A major concern when making line lists is the accuracy of the wavelengths, both relative and absolute. Iron lines were the standards for many years, but they suffer from being irregularly distributed across the spectrum and are of widely differing intensities. Harrison and others pushed for using thorium lines as standards because they are nearly evenly distributed, their intensities are reasonably uniform, and there are lots of them. As ruled gratings improved, recorded line shapes became more symmetrical, and the standards for accuracy rose even higher. We kept a list of those thorium lines which consistently gave smooth curves for interpolating wavelengths. There was still little hope of measuring line shapes even with the best of gratings - who has ever seen subsidiary maxima of spectrum lines, even though every elementary text explains and illustrates their positions and intensities.

\subsection{Intensities and Equivalent Widths}

Initially intensities were of secondary importance except on a relative basis. We arbitrarily assigned 100 to the strongest lines in a band and 1 to the weakest, because diatomic molecular line intensities change slowly and predictably, without the extreme variations found in atomic spectra.

The measurement of line intensities and shapes and equivalent widths was tentative at best. On our semi-automatic comparator we had a photomultiplier to register transmitted light. The intensity scale was calibrated with a step weakener as the plates were being exposed. Strong lines and very strong lines and extraordinarily strong atomic lines all produced the same photomultiplier output, which was automatically registered when a line position was measured. Shapes and hence wavelengths changed with intensity because of ghosts, satellites, and "grass" resulting from errors in ruling. A line of maximum strength might be recorded with an intensity of 100 , but a line 1000 times stronger was also recorded as 100 , although it might have a width many times greater because of saturation and blooming of the emulsion.

\subsection{Comments}

Work proceeded as above for some years, although improvements in computers made our data reduction much simpler and allowed us to measure and process 
it at ever increasing rates. By the time tab cards (popularly known as IBM cards) became obsolete, we had processed a million lines, and had that many cards stored in our comparator room to prove it. After a time our research took a dramatic turn for the better because of a combination of developments in instrumentation. It was a change of far greater significance than going from concave gratings to interferometrically ruled plane gratings.

\section{Current Practices}

With the advent of the Fourier transform spectrometer at the National Solar Observatory at Kitt Peak, there came major improvements in measuring line positions, intensities, shapes, and equivalent widths. Most importantly, the instrumental function is specifically determinable and can be corrected for if necessary. Ghosts, satellites, and grass or their equivalents are negligible for practical purposes. With this change came digital data processing and the recording of spectra as sets of numbers rather than images on a photographic plate or traces on a chart recorder.

\subsection{The Spectrometer}

A few words about the FTS may be appropriate. A Fourier transform spectrometer is a scanning Michelson interferometer. The input must be light from a stationary source (no changes in spectral composition or amplitude over short time intervals), but the output is an interferogram rather than a frequency or wavelength spectrum. The interferogram must undergo a Fourier transformation in order to display the spectrum. A diffraction grating automatically takes a Fourier transform of the incoming radiation and displays it with a one-to-one correspondence of wavelength and angle of diffraction. Here, the FTS only maps optical frequencies into very much lower spatial frequencies, and we must compute the transform. All data are digitized at an appropriate sampling rate so the mathematics of data reduction is similar to that of continuous functions. With the digitization comes ease of interactive manipulation at the keyboard of a computer. The data and resulting spectrum can be displayed and modified at will, to extract all the inherent information.

The maximum path difference of the Kitt Peak instrument is one meter, making the resolution limit $0.010 \mathrm{~cm}^{-1}$ (with this change in instrumentation we switch from wavelengths to wavenumbers, so we speak of $\mathrm{cm}^{-1}$ rather than $\mathrm{nm}$, with small intervals specified as millikaysers; $1 \mathrm{mK}=0.001 \mathrm{~cm}^{-1}$. The instrument therefore has a resolving limit of $10 \mathrm{mK}$ ).

The wavenumber ranges depend on the available detectors and beam splitters. Practically speaking the observable spectrum extends from $400 \mathrm{~cm}^{-1}$ (25 $\mu \mathrm{m})$ to $40000 \mathrm{~cm}^{-1}(250 \mathrm{~nm})$ with three sets of beam splitters and detectors. All of our observations to date lie in the region $1800 \mathrm{~cm}^{-1}(5.5 \mu \mathrm{m})$ to 40000 $\mathrm{cm}^{-1}$. The time required for a single scan at maximum resolution is about 15 minutes. A minimum number of 4 scans is desirable because it reduces the noise by a factor of 2 . 


\subsection{Light Sources}

All light sources used with the FTS must be steady in intensity and spectral composition, with no sudden changes on a time scale consistent with the spectral range being observed. Just think of the Fourier transform of a sudden change. If the transform has frequencies in the range being observed, then the source is unsatisfactory. Let's consider some examples. A sinusoidal modulation, such as occurs when a power supply is insufficiently filtered or a plasma is oscillating, produces sidebands on every spectral line.

Sharp spikes in intensity, which frequently and irregularly occur in many sources, produce a broad noise spectrum. A dramatic illustration of this is found in the use of a furnace for infrared spectra. With something like $\mathrm{ZrO}_{2}$ as a charge, when it melts, small hot particles are produced. These fly around inside the furnace until steady-state conditions are reached, and sometimes cling to the top of the bore, where they come loose at irregular intervals for quite some time. As they pass across the bore, they cross the region imaged on the spectrometer entrance aperture and cause large fluctuations in the observed intensity. Hot particles are bright in the infrared! The result is lots of noise in the transformed spectrum from these spikes in intensity.

In spite of these problems, for emission spectra we have had good success with use of several sources: the furnace, discharge tubes both sealed and unsealed and with and without electrodes, hollow cathodes, inductively coupled plasmas, diffusion flames, and a carbon arc (once or twice).

For absorption spectra, the problems usually have nothing to do with the stability of the background source but instead with its spectral composition and emissivity, as will be made clear below with a specific example. The "blackbody" temperature of a lamp may be $2500 \mathrm{~K}$ in the visible region of the spectrum, but decrease to $1800 \mathrm{~K}$ in the near infrared. When something like the sun is used for background radiation, the spectrum with the laboratory absorber in place must be ratioed with the source spectrum alone.

\subsection{Data Reduction and Analysis, Emission Spectra}

Raw data are written onto a magnetic tape, and then transformed and placed in a format which is used by the code DECOMP (Brault \& Abrams 1989). It is an IBM PC compatible interactive data processing code especially for input from the NSO Kitt Peak FTS (it can, however, be adapted easily for use with spectral data obtained from any kind of instrument). The picking of branches and the subsequent analysis is accomplished with the help of the code ANALYSIS (Pecyner \& Davis 1988).

The whole process goes something like this: The transform of the FTS interferogram is a set of numbers representing the signal strength at equally spaced points on the wavenumber axis. In turn we (1) plot them at low resolution to take a first look at the spectrum; (2) subtract the background; (3) make a low resolution atlas to get a perspective of the entire system, especially the largescale features such as band heads; (4) make atlases at full resolution, both low 
and high dispersion; (5) pick the lines; (6) fit the lines; (7) prepare a finished line list; (8) pick the branches; (9) calculate the molecular parameters; (10) calculate FC factors and potential curves;

To make this sequence clearer let's follow it through each step with examples from our recent or current work. Let's start with how we treat the data after they are transformed.

Fig. 2 shows a low-resolution plot of the transformed data for $\mathrm{FeH}$, to illustrate the need for background subtraction. The spectrum comes from a carbon tube furnace charged with iron, hydrogen, and helium. The data, consisting of about two million points, were separated into 4096-point sections and the points in each section averaged to make this plot.

Fig. 3 shows a plot of the minima in each of the sections. This curve is smoothed and filtered as necessary, stretched to cover the entire original set of data points, and then subtracted from the data.

Fig. 4 is a low-resolution plot of the background-subtracted data. These three plots are linear in the intensity scale.

Fig. 5 shows a full resolution plot with low dispersion. The intensity scale is linear up to 10 , and logarithmic thereafter.

Fig. 6 shows part of a full resolution plot with high dispersion. The scale is linear $/ \log$ as in the previous Figure.

Fig. 7 shows the same spectral lines, this time on a linear scale, whose positions have been located by our line-finding program. A line is located by finding the slopes at the points where the second derivatives of the data are zero, determining where the tangents cross, and averaging this position with that of the maximum of the data for the line. This position is only a first approximation. Lines must always be fitted in order to obtain accurate measures of their positions, intensities, and widths, because there is only a limited number of sampling points in each line, and a sampling point does not generally coincide with the peak of a line. The lower part of the Figure shows residuals after the lines are fitted and the fitting function subtracted. For the fitting, the model of a Voigt profile is chosen. This profile has gaussian and lorentzian components. The program performs a non-linear least-square fit, and adjusts the intensity, position, width, and ratio of gaussian to lorentzian component, in order to get the best fit. Any of these parameters can be fixed when it is appropriate to do so.

Fig. 8 shows a magnified version of the residuals. It is evident that there is an oscillating component to the spectrum which has not been accounted for. This oscillation is called ringing, and is visible at the feet of sharp spectral lines - lines narrower than the resolving limit of the spectrometer. With proper filtering and fitting, the ringing can be properly removed and the line restored to its original shape as emitted by the source. The process is not the same as simply smoothing the data to hide the oscillations, but has a sound basis in information theory.

Fig. 9 shows the numerical values of the molecular parameters for each line. The intensities are accurate to a few percent, widths and equivalent widths to a few percent, and position to an accuracy depending on the signal-to-noise ratio. In broad terms it has a wavenumber precision as good as the experimental 


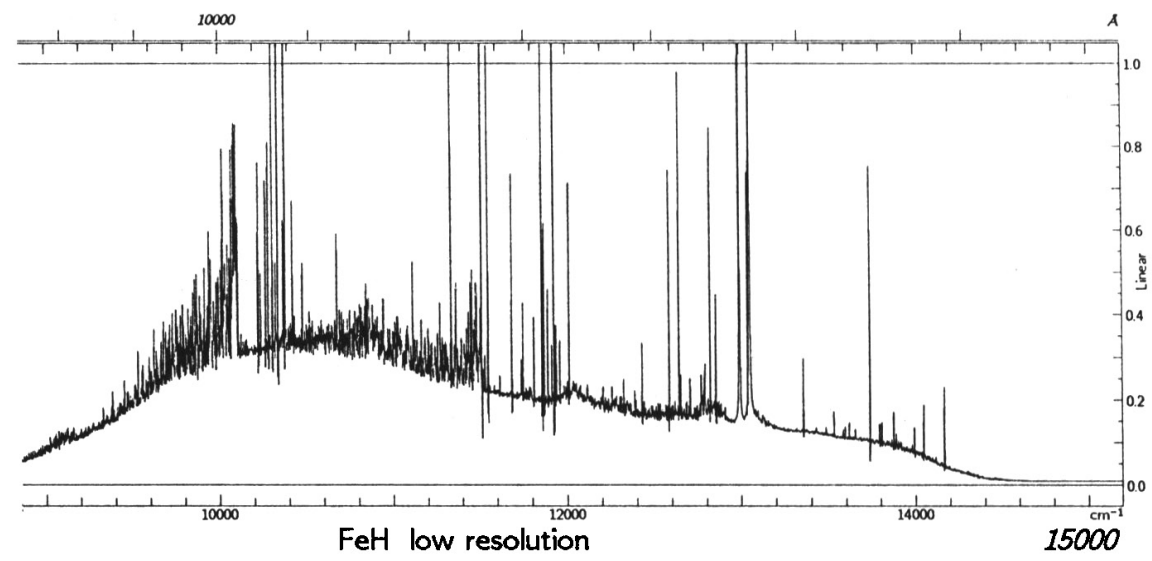

Fig. 2. Low resolution plot of the FeH furnace spectrum from 9000 to $15000 \mathrm{~cm}^{-1}$.

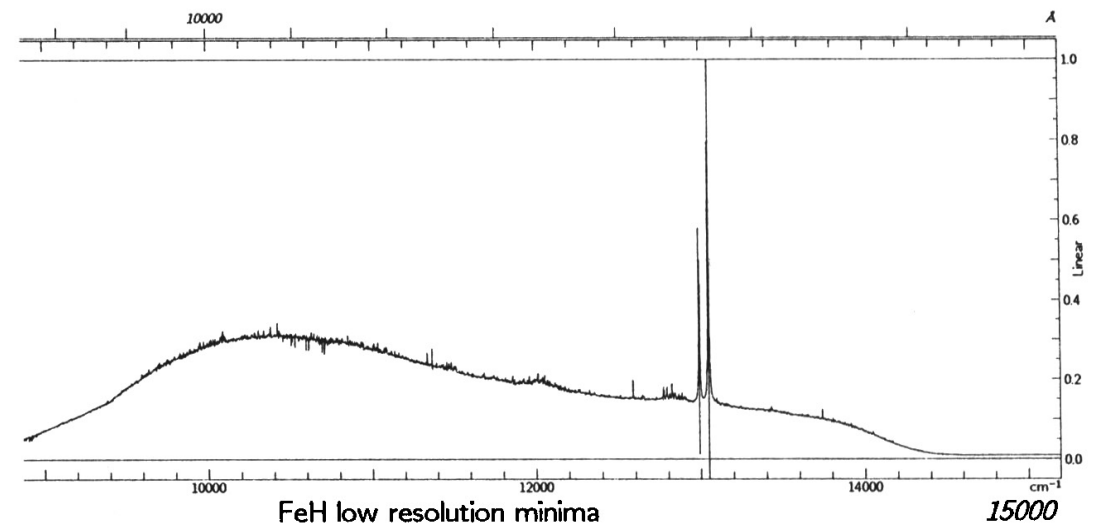

Fig. 3. Plot of minima in the low resolution $\mathrm{FeH}$ spectrum.

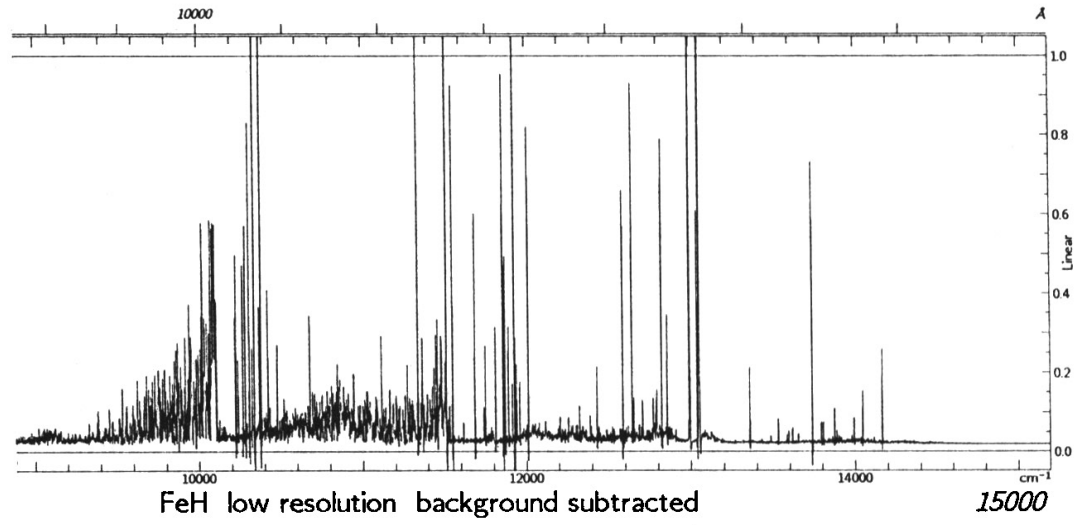

Fig. 4. Background-corrected low resolution FeH spectrum 


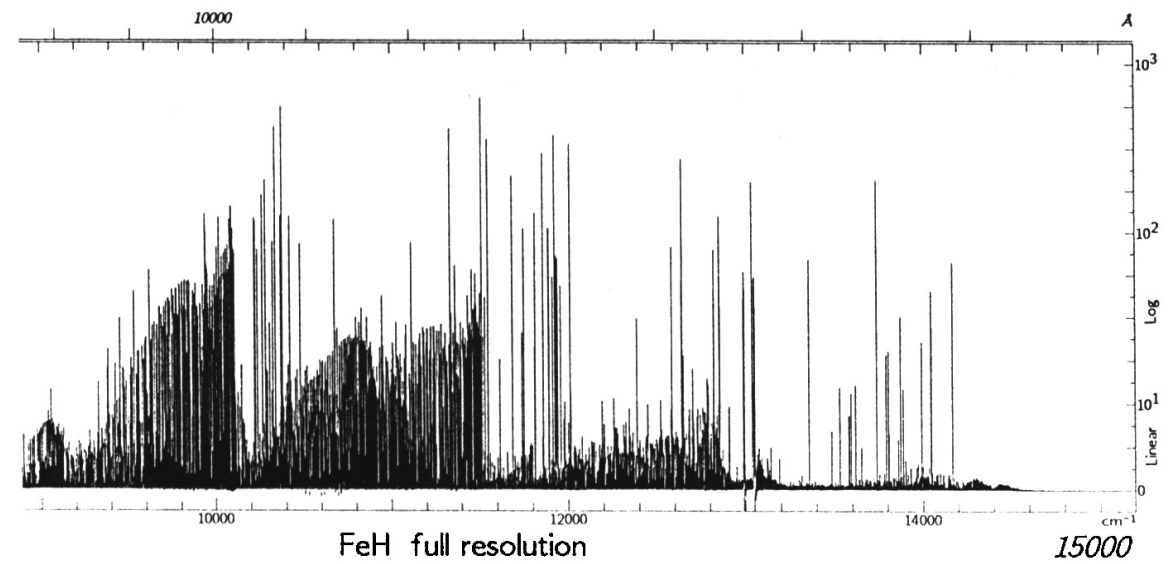

Fig. 5. Full-resolution plot of background-corrected FeH spectrum. Note that weak features are enhanced because the intensity scale is logarithmic above 10

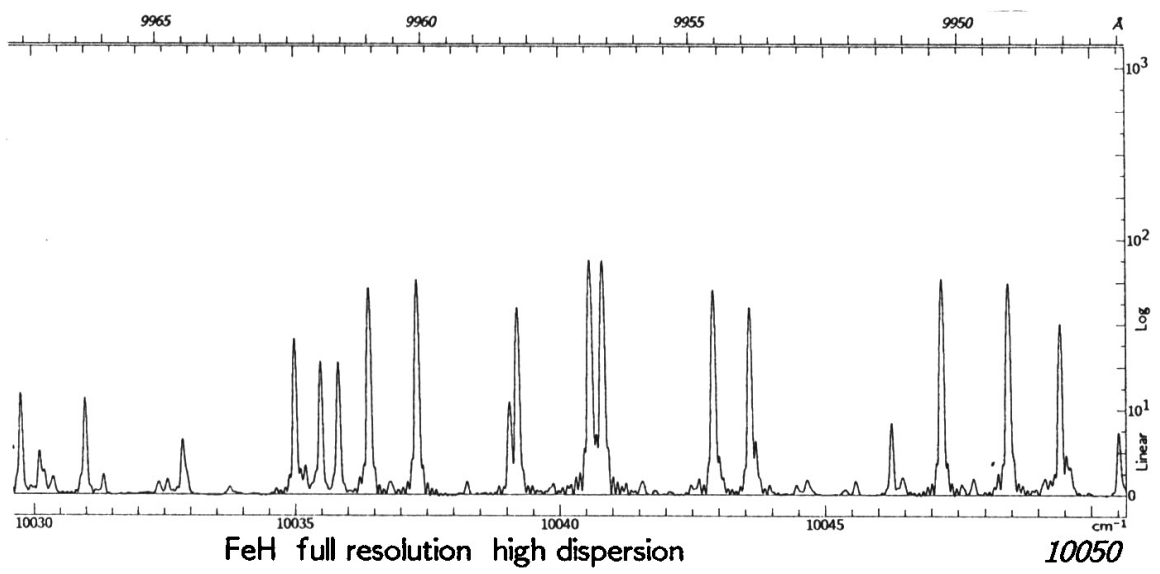

Fig. 6. FeH spectrum plotted at full resolution with high dispersion

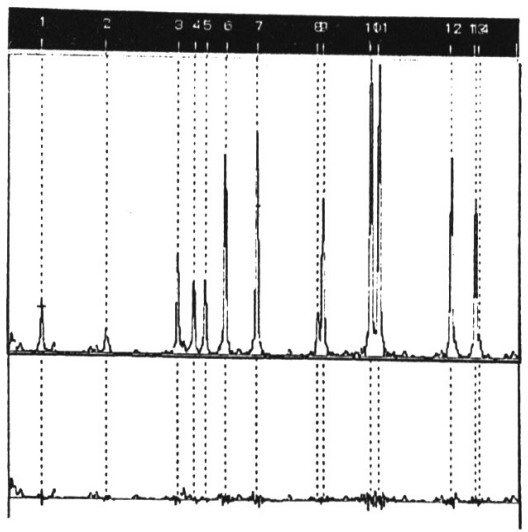

Fig. 7. (upper) FeH spectrum with lines picked by a line-finding program. (lower) Residuals after fitting and subtracting the fitting function (Voigt profile) 


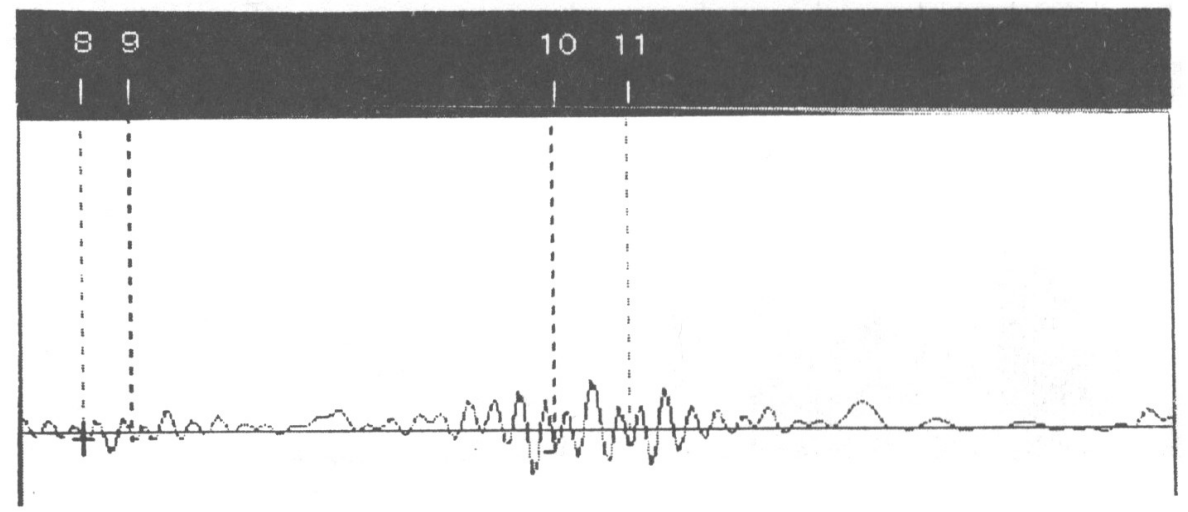

Fig. 8. Magnified residuals showing spectrum line "ringing".

$\begin{array}{rcccccc}\text { No. } & \text { Sigma } & \text { Amp } & \text { Wid(mK) } & \text { Dmp } & \text { Itn } & H \\ 1 & 10030.9690 & .0355 & 76.4 & .664 & 8 & 0 \\ 2 & 10032.8438 & .0189 & 99.4 & .750 & 10 & 0 \\ 3 & 10034.9711 & .0798 & 69.3 & .507 & 6 & 0 \\ 4 & 10035.4643 & .0578 & 74.0 & .572 & 5 & 0 \\ 5 & 10035.8026 & .0569 & 74.5 & .404 & 5 & 0 \\ 6 & 10036.3877 & .1586 & 72.0 & .314 & 4 & 0 \\ 7 & 10037.2975 & .1772 & 71.6 & .299 & 5 & 0 \\ 8 & 10039.0365 & .0328 & 81.1 & .000 & 10 & 0 \\ 9 & 10039.1883 & .1214 & 74.6 & .273 & 9 & 0 \\ 10 & 10040.5597 & .2301 & 74.8 & .314 & 5 & 0 \\ 11 & 10040.7993 & .2280 & 74.7 & .311 & 3 & 0 \\ 12 & 10042.8837 & .1550 & 72.5 & .397 & 7 & 0 \\ 13 & 10043.5663 & .1219 & 70.8 & .303 & 12 & 0 \\ 14 & 10043.6822 & .0153 & 55.8 & .945 & 13 & 0\end{array}$

\begin{tabular}{|c|c|c|c|c|}
\hline $\mathbf{T}$ & Ew & rms & even & odd \\
\hline L & 3.76 & .0011 & .0007 & .0009 \\
\hline L & 2.70 & .0011 & .0002 & .0011 \\
\hline$\overline{\mathrm{L}}$ & 7.22 & .0023 & .0023 & .0012 \\
\hline I & 5.73 & .0018 & .0010 & .0014 \\
\hline 工 & 5.31 & .0010 & .0007 & .0007 \\
\hline L & 13.80 & .0027 & .0025 & .0009 \\
\hline L & 15.24 & .0030 & .0029 & .0009 \\
\hline $\mathrm{L}$ & 2.84 & .0015 & .0012 & .0009 \\
\hline I & 10.78 & .0017 & .0015 & .0008 \\
\hline L & 20.79 & .0043 & .0040 & .0017 \\
\hline L & 20.57 & .0042 & .0038 & .0016 \\
\hline L & 14.05 & .0031 & .0027 & .0018 \\
\hline I & 10.38 & .0018 & .0017 & .0008 \\
\hline r & 1.32 & .0017 & .0011 & .0013 \\
\hline
\end{tabular}

Fig. 9. Line parameters resulting from the fitting process. "Amp is the amplitude on an arbitrary scale, "Wid $(\mathrm{mK})$ is the width at half maximum in units of $10-3 \mathrm{~cm}^{-1}$ (millikayser), "Dmp" is the damping constant - 0 is pure gaussian profile, 1 is pure lorentzian, "Itn" is the number of iterations in the fitting, " $H$ " and "T" are informational columns, "Ew" is the equivalent width (area), "rms", "even", and "odd" are the residuals of the fit

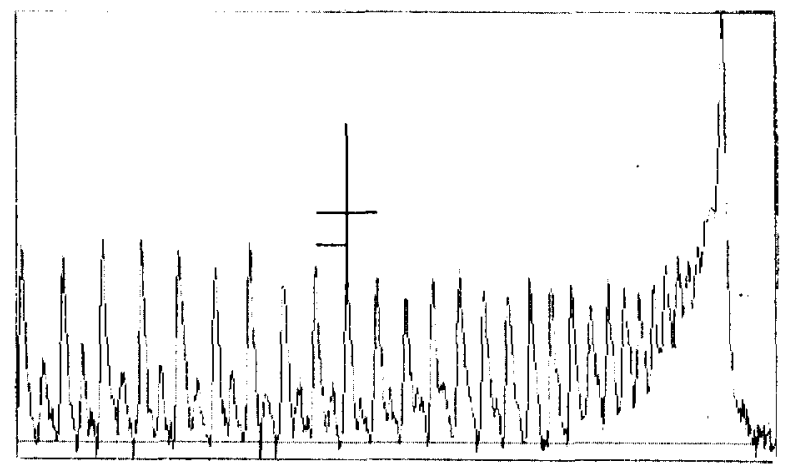

Fig. 10. (0-0) band head of CaS at $15200 \mathrm{~cm}^{-1}$, degraded to the red. 
repeatability of the source's optical positioning and its operating conditions. In many cases this is a few tenths of a $\mathrm{mK}$. For the absolute calibration of wavenumbers, a first approximation is taken from the wavenumber of the laser which controls the position of the mirrors. It is temperature stabilized, and in practice the repeatability is a few $\mathrm{mK}$. A few standard lines from argon or CO or other species at any convenient wavenumber are sufficient to set absolute standards everywhere in the spectrum. From this file we construct a line list.

Fig. 10 shows this bandhead, degraded to the red (left). Now we take the linelist and plot it, instead of plotting the full spectrum profile. We obtain a histogram, or "stick" spectrum, as shown in Fig. 11. We can easily see the branches, and assign a few lines; these lines are then extended by extrapolation, more picked, and the process proceeds. The numbers at the top are the coefficients of the fit, and the last one gives an idea of how important the last term is. "Leaf" refers to which branch the line at the cursor belongs; "Number" is the running number $m$ in the polynomial fit, equal to $-\mathrm{J}$ for P-branches and $\mathrm{J}+1$ for $\mathrm{R}$ branches; "Sigma" is the wavenumber in $\mathrm{cm}-1$, and "Weight" tells whether the line is included in the fit or not (lines more than $3.2 \sigma$ standard deviations).

With a few more keystrokes the molecular parameters are calculated, as shown in Fig. 12. The covariance-correlation matrix is also printed out, as are data on the spectrum lines. Column 1 is the running number $\mathrm{m}$, column 2 is the wavenumber in $\mathrm{cm}^{-1}$, column 3 is the intensity, and column 4 tells whether the line is included in the purged fit.

Finally, calculation of Franck-Condon factors is routine, accomplished with another code (Jarmain \& McCallum 1970).

\subsection{Data Reduction and Analysis, Absorption Spectra}

Data reduction and analysis for absorption spectra is very little different from that for emission spectra, except for initial treatment of the data. The background is no longer subtracted from the spectrum, but ratioed with it. The next step is to take the logarithm to transform the data to an absorbance spectrum. From then on the procedures are identical. Let's look at the solar spectrum to illustrate the ratioing process.

Fig. 13 is a section of the solar spectrum observed from Spacelab 3, from 2000 to $2500 \mathrm{~cm}^{-1}$ in the infrared. The plot has been normalized so that the top of the plot is 1.0 . The bottom is arbitrarily set to 0.4 The bandheads of a $\mathrm{CO}$ sequence are quite evident, as is the fact that the background radiation is far from constant in intensity. As a result, the plot does not truly represent the relative intensities of the lines. Instead, they are given by the ratio of the depth of each peak from the back ground, to the distance from zero intensity to the background, and consequently a background correction by ratioing is required.

Fig. 14 shows the same spectrum with the background straightened. A casual glance might indicate that the atlas is now an accurate representation of widths and intensities. Evidently some of the early atlases were prepared in just this way. 


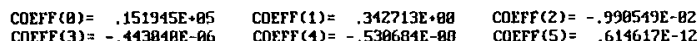
STD RESIDUE $=$ Q255

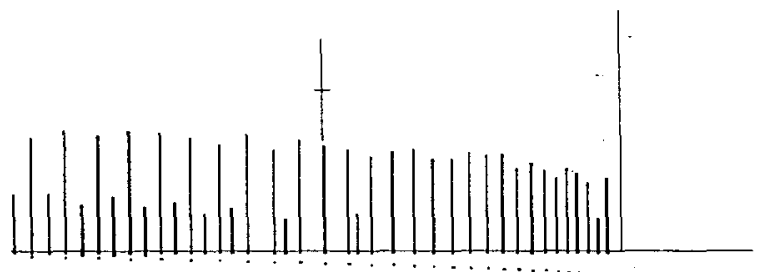

Fig. 11. Histogram of (0-0) band of CaS with $P$ and $R$ branches marked.

\begin{tabular}{|c|c|c|c|c|}
\hline \multicolumn{5}{|c|}{$\begin{array}{l}\text { MODEL NUMBER \& DEGREE OF FIT }=5 \\
\text { RESULTS AFTER PURGING }\end{array}$} \\
\hline $\begin{array}{l}V O= \\
B^{\prime}= \\
D^{\prime}= \\
H^{\prime}= \\
B^{\prime \prime}= \\
D^{H}=\end{array}$ & $\begin{array}{r}15194.4336 \\
.16648921 \mathrm{E}+0 \\
.12594174 \mathrm{E}-\mathrm{C} \\
.58787490 \mathrm{E}- \\
.17638233 \mathrm{E}+\mathrm{C} \\
.11989182 \mathrm{E}-\mathrm{C}\end{array}$ & $\begin{array}{l}61+1- \\
00+1- \\
06+1- \\
12+1- \\
00+1- \\
06+1-\end{array}$ & $\begin{array}{l}.0009 \\
.98 E-0 \\
.99 E-0 \\
.31 E-1 \\
.98 E-0 \\
.98 E-0\end{array}$ & $\begin{array}{l}96 \\
05 \\
09 \\
13 \\
05 \\
09\end{array}$ \\
\hline m & $\begin{array}{l}\text { TANDARD RESIDU } \\
\text { Bigma }\end{array}$ & ${ }_{\text {int }}=$ & $\begin{array}{c}.0058 \\
\text { fit }\end{array}$ & residual \\
\hline $\begin{array}{l}-176.0 \\
-173.0\end{array}$ & $\begin{array}{l}14824.63078 \\
14836.20041\end{array}$ & $\begin{array}{l}.1614 \\
.1796\end{array}$ & $\begin{array}{l}0 . \\
0 .\end{array}$ & $\begin{array}{l}-.71227 \\
-.59507\end{array}$ \\
\hline $\begin{array}{l}-125.0 \\
-124.0\end{array}$ & $\begin{array}{l}14996.37290 \\
14999.21302\end{array}$ & $\begin{array}{l}.4831 \\
.5627\end{array}$ & $\begin{array}{l}1 . \\
1 .\end{array}$ & $\begin{array}{l}-.00274 \\
-.00853\end{array}$ \\
\hline $\begin{array}{l}125.0 \\
-91.0 \\
124.0\end{array}$ & $\begin{array}{l}15080.36917 \\
15081.24413 \\
15082.56551\end{array}$ & $\begin{array}{l}.4596 \\
.6392 \\
.3696\end{array}$ & $\begin{array}{l}\text { 0. } \\
\text { 1. } \\
\text { 1. }\end{array}$ & $\begin{array}{r}.01359 \\
-.00320 \\
.00310\end{array}$ \\
\hline $\begin{array}{l}25.0 \\
23.0 \\
\text { llead }\end{array}$ & $\begin{array}{l}15196.86401 \\
15197.05039\end{array}$ & $\begin{array}{r}.1537 \\
.3449\end{array}$ & $\begin{array}{l}0 . \\
0 .\end{array}$ & $\begin{array}{r}-.05182 \\
.02815\end{array}$ \\
\hline
\end{tabular}

Fig. 12. Molecular parameters. Only a few of the lines are shown, to illustrate the branches: $-\mathrm{m}=\mathrm{J}$ for $\mathrm{P}$ branches, $\mathrm{M}=\mathrm{J}+1$ for $\mathrm{R}$ branches.

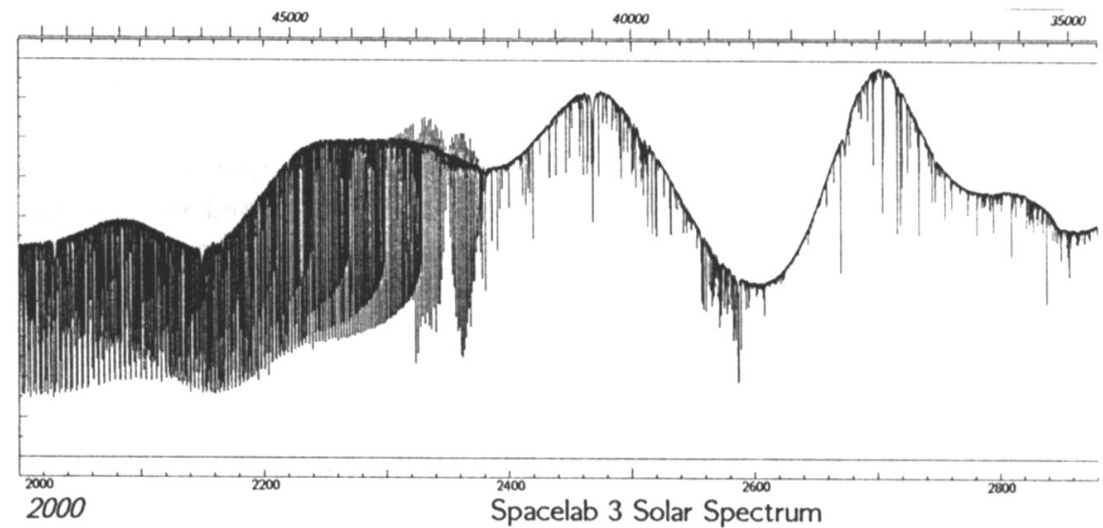

Fig. 13. A portion of the solar spectrum as observed from Spacelab 3. The intensity scale on the vertical axis is linear from .4 to 1.0 


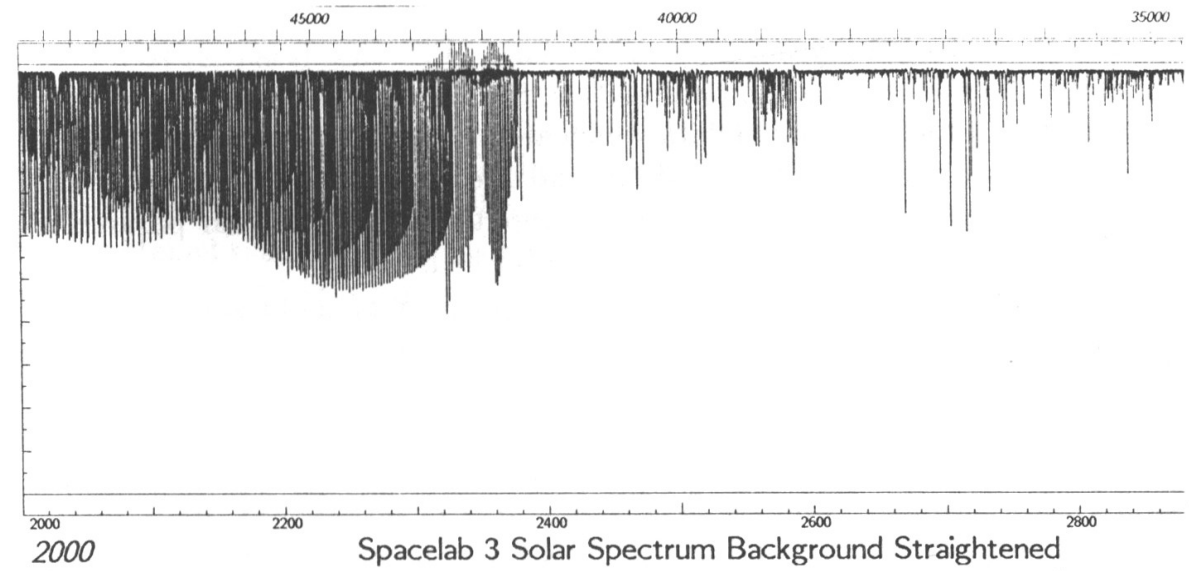

Fig. 14. Spacelab 3 solar spectrum with the background arbitrarily set to 1.0. The intensity scale is linear from .4 to 1.0

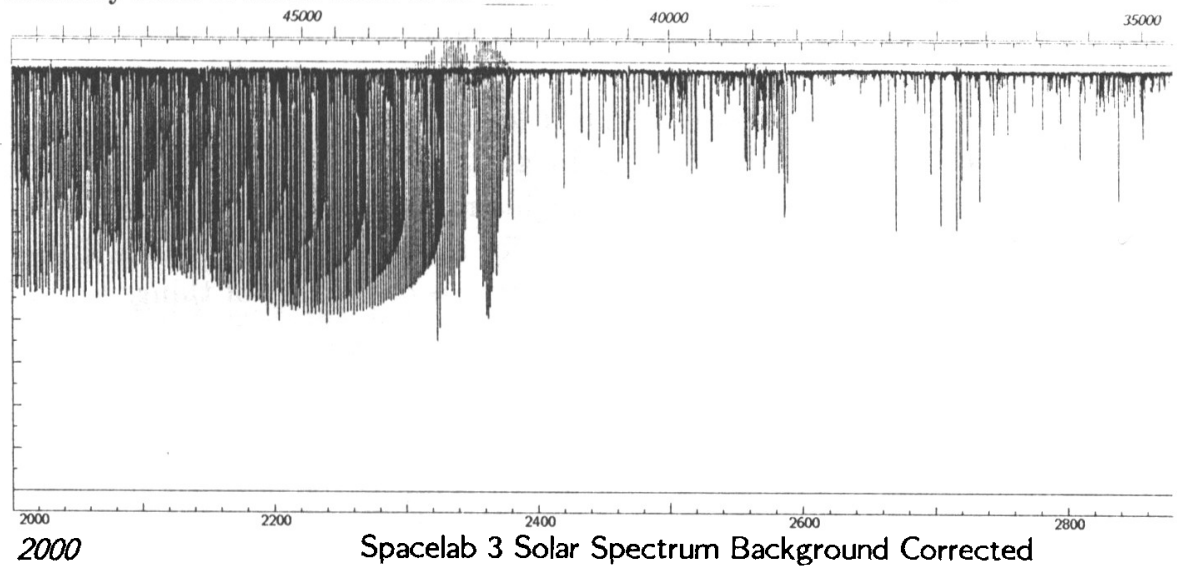

Fig. 15. Spacelab 3 solar spectrum with the background arbitrarily set to 1.0. The intensity scale is linear from .4 to 1.0

However, a look at Fig. 15 which is the correctly ratioed spectrum, shows this is not the case. In the region near $2000 \mathrm{~cm}^{-1}$, for example, true intensities are about one-third greater than the background-straightened spectrum shows. The gradual and monotonic changes in intensities give a clue that the representation is now accurate.

When we take the logarithm of this spectrum, plot it, and pick and fit the lines as before, we then have the true intensities and equivalent widths from which we can make further calculations.

\subsection{Line Lists, Analyses, and Atlases}

From time-to-time we get requests for line lists. We are happy to provide them when we are able, and will often make up something special. However, it has 
only been the last several years since the advent of PCs that it is easy to prepare them and write the information on diskettes. Earlier work is occasionally stored on paper, except for the original data which are on magnetic tape. Anything unpublished almost always has to be redone, and of course ever after is then readily available. The same is true for analyses - computation of molecular parameters - but in these cases all our work has been published.

We also get requests for atlases geared to a specific region of a spectrum, with a specified dispersion. These are fun to answer because it means someone has a pressing astronomical problem which needs resolution, and maybe we can help. Let me tell you of one we are working on now. Prof. Jesse Greenstein needs a spectrum of VO to compare with the stellar spectrum of GD165B, "the faintest, coolest known M-dwarf", and the next coolest star PC0025+0447. He suggested that I look at a paper (Kirkpatrick et al. 1993) to compare spectra. On my next trip to Kitt Peak I charged the electric furnace with vanadium oxide and took a spectrum from 8000 to $20000 \mathrm{~cm}^{-1}$, from which I prepared atlas plots to match the spectrum presented in the paper referenced above.

Fig. 16 shows a comparison. Greenstein's comment was, "If I blur my eyes and stand on my head, I would think that it looks about right". I have inverted the emission spectrum in this figure, thereby avoiding the need to stand on one's head. Then he goes on to suggest how I might translate my emission spectrum into an absorption spectrum with saturation, for a possible better match. Other tasks have absorbed my time, but the translation is on my list of things to do.

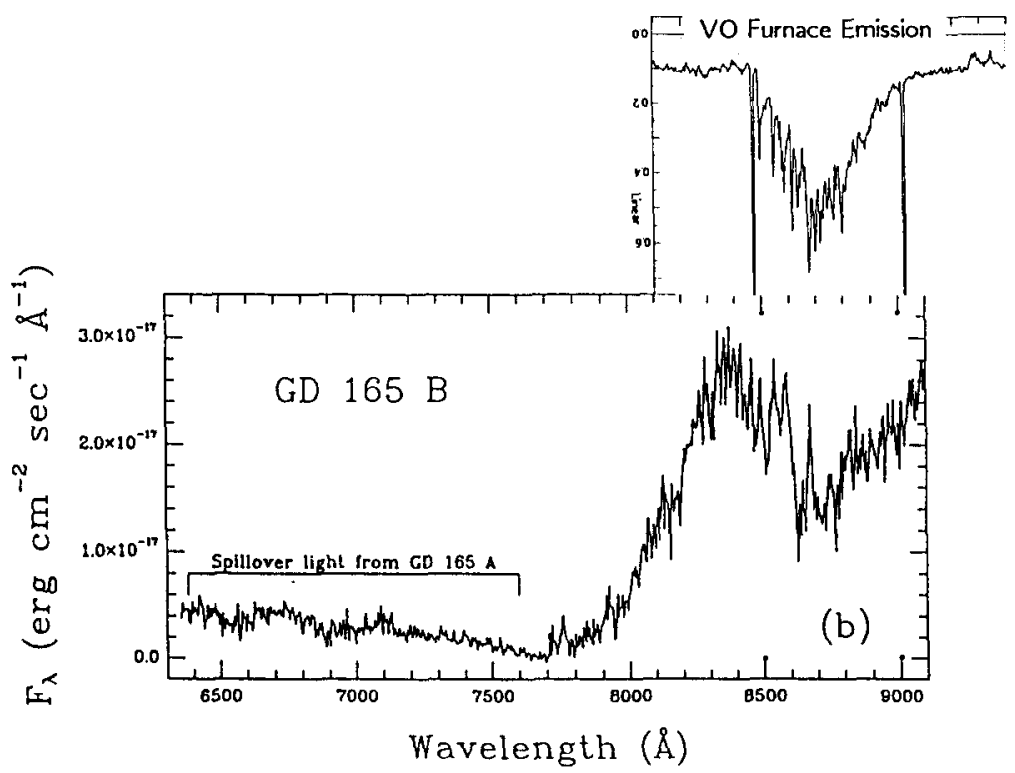

Fig. 16. Absorption spectrum from GD165B (Kirkpatrick et al. 1993) compared with VO furnace emission spectrum (inverted) 


\subsection{Comments}

What's next? Our three most recent publications are transition rates from absorption measurements in ZrO (Littleton et al. 1993), an unidentified band in the infrared spectrum of FeH (Phillips \& Davis 1993), and the infrared spectrum of the Meinel system of $\mathrm{OH}$ (Abrams et al. 1993). For current work, the complex spectrum of FeH in the green centered on $530 \mathrm{~nm}\left(18400 \mathrm{~cm}^{-1}\right.$ to $\left.19400 \mathrm{~cm}^{-1}\right)$ is being extensively studied. There are over 1000 lines in this region strong enough to merit assignment to rotational subbands, but there are no obvious subband heads or any obvious rotational series. A program searching for $\mathrm{P}$ and $\mathrm{R}$ branch pairs has found four groups of red-degraded subbands with heads in the regions $18800,18970,19170$, and $19360 \mathrm{~cm}^{-1}$. There is no evidence of $Q$ branches. The structure of the branches is quite compatible with an assignment to FeH. If this preliminary assignment proves correct, the carrier is an $\mathrm{FeH}$ transition with delta-lambda equal to zero. Only about one-quarter of the 1000 plus lines in this region have been assigned to any branch.

We have an abundance of data for CN from every known source, and have put them in the hands of M.L.P. Rao (Andhra University) for unraveling the perturbations and making a global fit. We are completing our analysis of $\mathrm{CaS}$; we are trying to unravel why $f$-values in the CaH spectrum are different for $P$ and $R$ branches; and we are thinking about new solar data from the space shuttle.

For the time being $\mathrm{LaS}, \mathrm{LaO}, \mathrm{YS}$, and $\mathrm{ZrS}$ are dormant, although we have data for them all.

\section{References}

Abrams M.C., Davis S.P., Rao M.L.P., Engleman R. Jr., Brault J.W., 1993, Astrophys. J. Suppl., submitted

Brault J.W., Abrams M.C., 1989, Opt. Soc. Am. Tech. Digest, 6, 120

Davis S.P., 1987, Publ. A.S.P., 99, 1105

Davis S.P., Phillips J.G., 1963, The Red System of the CN Molecule, (Berkeley: University of California Press)

Gatterer A., Junkes J., Salpeter E.W., Rosen B., 1957, Molecular Spectra of Metalic Oxides, Specola Vaticana

Jarmain W.R., McCallum J.C., 1970, TRAPRB: a computer program for molecular transitions, (Physics Department, Univ. Western Ontario, Canada)

Kirkpatrick J.D., Henry T.J., Liebert J., 1993, Astrophys. J., 406, 701

Littleton J.E., Davis S.P., Song M., 1993, Astrophys. J., 404, 412

Pecyner R., Davis S.P., 1988, Appl. Opt. 27, 3775

Phillips J.G., 1959, Astrophys. J., 130, 308

Phillips J.G., Davis S.P., 1962, J. Opt. Soc. Am., 52, 480

Phillips J.G., Davis S.P., 1968, The Swan System of the $C_{2}$ Molecule, (Berkeley: University of California Press) 